\title{
Prophylactic central lymph node dissection in differentiated thyroid cancer
}

\author{
Julie A. Goddard · David L. Steward
}

Received: 21 March 2013/ Accepted: 2 May 2013/Published online: 23 May 2013

(C) Springer Science+Business Media New York 2013

Conzo et al. [1] describe their experience of treating differentiated thyroid carcinoma in patients who lack clinically evident nodal disease (cN0) with total thyroidectomy, without prophylactic central lymph node dissection, followed by radioactive iodine (RAI). $93.8 \%$ of their 221 patients had AJCC Stage I or II papillary thyroid carcinoma. They report a low locoregional recurrence rate of $3.16 \%$ after almost 10 years as well as low complication rates $(0.91 \%$ for both permanent hypoparathyroidism and permanent vocal fold paralysis). The merits of prophylactic lymph node dissection are debated in these patients with early-stage disease, and the authors' discussion highlights this debate. They note that the current literature lacks conclusive evidence of survival or recurrence benefit, but has established an increase in complication rates (specifically, transient hypocalcemia) associated with prophylactic central nodal dissection compared with total thyroidectomy alone. Based on their results and their scrutiny of this literature, those authors have come to conclude that their treatment paradigm is acceptable and that prophylactic central neck dissection can be avoided [1].

Multiple staging systems for papillary thyroid carcinoma have been developed, many of which do not include cervical nodal metastasis as a factor. However, TNM staging is utilized by the American Thyroid Association in its most recently published guidelines for differentiated thyroid carcinoma. As with other cancers, this system includes clinical staging as well as pathologic staging for nodal metastases. Patients who do not have suspicious

J. A. Goddard $(\square)$. D. L. Steward

Department of Otolaryngology-Head and Neck Surgery,

University of Cincinnati College of Medicine, P.O. Box 670528,

231 Albert Sabin Way, Cincinnati, OH 45267-0528, USA

e-mail: julie.goddard@uc.edu cervical lymph nodes by preoperative palpation, preoperative ultrasound, or intraoperative inspection are designated clinically node negative, or cN0. If there are lymph nodes with suspicious characteristics by these evaluations, then patients are considered clinically node positive, or $\mathrm{cN} 1$. Pathologic staging is based on microscopic evaluation of nodes in a surgical specimen. Patients without pathologic evidence of nodal disease are staged $\mathrm{pNO}$ and those with disease are designated $\mathrm{pN} 1$.

The importance of clinical nodal evaluation is evident in the article by Conzo et al. Patients are appropriately classified as $\mathrm{cNO}$ versus $\mathrm{cN} 1$, as evidenced by the exclusion of 211 patients who had clinically suspicious lymph node findings by preoperative ultrasound or intraoperative inspection. These $\mathrm{cN} 1$ patients underwent therapeutic central nodal dissection rather than prophylactic, and this is a critical distinction. Benefit of central compartment dissection in the setting of clinically positive nodes is widely agreed upon. However, definitive evidence regarding oncologic benefit from dissecting clinically negative nodal basins has yet to be established, and the significance of microscopic nodal disease is widely questioned. A closer look at nodal metastases was recently provided the ATA [2]. In this publication, Randolph et al. note the differences in recurrence risk in node-positive patients based on nodal characteristics. Specifically, cN0 patients who have low volume microscopic central nodal metastases without extranodal extension in neck dissection specimens (making them $\mathrm{pN} 1$ ) still have very low recurrence risk (median $2-4 \%$ which is similar to recurrence risk in $\mathrm{cN} 0$ patients in whom nodal dissection is not performed). This is compared with a $22 \%$ recurrence risk in patients who are cN1. Though final TNM nodal staging of these two groups of patients is the same, N1, the marked difference in recurrence between $\mathrm{cNO}$ and $\mathrm{cN} 1$ patients highlights the 
importance of identification of clinically positive nodes before and during surgery, and our ability to identify these positive nodes accurately and consistently. Shen et al. [3] similarly noted different recurrence rates in $\mathrm{cNO}$ versus $\mathrm{cN} 1$ patients (no prophylactic dissections performed), concluding that surgeon's assessment of the central neck could accurately predict those who benefit from central nodal dissection. Conzo et al. can be applauded for their accurate clinical assessment of the central neck; their $3 \%$ recurrence rate is similar to other such reports on $\mathrm{cN} 0$ patients.

The vast majority $(95.47 \%)$ of the patients in the publication by Conzo et al. underwent adjuvant RAI. These patients were treated before 2005 during a time when RAI was perhaps used more liberally and before the emergence of recent trends to limit its usage. RAI has been accepted as a component of treatment for differentiated thyroid cancer for more than five decades. RAI may be employed for ablation and/or treatment. Ablation refers to use of RAI to destroy any residual thyroid tissue remaining after thyroidectomy, whereas treatment refers to subsequent use of RAI when evidence of residual thyroid tissue or residual/ recurrent disease is present [11]. The routine usage of postoperative RAI for ablation purposes in all patients with differentiated thyroid carcinoma is under scrutiny, specifically in patients considered to have low-risk disease. Similarly, optimal dosing of RAI is a question not yet settled. Wartovsky and Van Nostrand [11] discuss RAI treatment for well-differentiated thyroid cancer in their 2012 review, including objectives, indications, and dosing strategies. They discuss proposed objectives for RAI ablation including (1) ablation of residual thyroid tissue which subsequently allows for improved sensitivity of surveillance whole-body radioiodine scans for detection of recurrence and facilitates interpretation of follow-up serum thyroglobulin levels; (2) treatment of residual postoperative microscopic tumor foci; (3) decreased recurrence; and (4) increased survival. These objectives are summarized from both the ATA guidelines and European Consensus. However, Wartovsky points out recent literature related to these objectives in patients with low-risk differentiated thyroid cancer. This literature includes a 2012 retrospective study indicating that RAI ablation did not significantly impact survival/recurrence in low-risk patients and another large 2012 randomized prospective trial demonstrating equivalence of low dose RAI $(30 \mathrm{mCi})$ and higher dose $(100 \mathrm{mCi})$ at achieving successful postoperative ablation in low-risk patients. Further studies are in progress regarding avoidance of RAI in low-risk patients. Wartovsky [11] notes that indications for RAI usage are narrowing, with trends toward lower dosages for ablation and more patients not undergoing ablation at all. The debate regarding optimizing indications and dosing strategies for RAI in low-risk patients parallels the debate regarding surgical strategies in cN0 patients and, indeed, studies regarding one issue may help in answering questions regarding the other.

Conzo et al. [1] observe that for cN0 patients in whom the decision to give RAI is made preoperatively, prophylactic central lymphadenectomy adds no benefit. While advocates for routine prophylactic nodal dissection have not yet proven definitive recurrence/survival benefit, some propose value added regarding diminished thyroglobulin (TG), suggesting it as a surrogate for recurrence, and more accurate staging which can guide adjuvant RAI usage. In 2006, Sywak et al. [4] reported lower TG levels in patients who underwent prophylactic central dissection measured before RAI ablation and 6 months after ablation compared with a historical control group undergoing total thyroidectomy alone followed by RAI ablation. This research group expanded their study to multiple centers in a more recent publication, but the lower TG seen 6-12 months after RAI ablation in the patients undergoing central dissection was no longer statistically significant [5]. Whether lower levels of TG can be truly indicative of clinically significant diminished recurrence is debatable. In a similar type of comparison study, Hughes et al. [6] found no difference in TG, but they noted an upstaging of almost one third of their $\mathrm{cN} 0$ patients older than 45 to Stage III disease based on pathologically positive central nodes (pN1a), which subsequently affected the dose of postoperative radioactive iodine ( $150 \mathrm{mCi}$ for $\mathrm{pN} 1$ vs. $30 \mathrm{mCi}$ for $\mathrm{pN} 0$ ). Similar upstaging of patients had previously been noted by Shindo et al. [7]. Studies have yet to clarify whether this upstaging translates into identification of truly higher risk patients who may benefit from more aggressive RAI dosing, or whether these upstaged patients are overtreated.

The opposite side of this issue is that pathologic information from central nodal pathologic data in $\mathrm{cNO}$ patients may allow us to less aggressively dose RAI, or completely avoid it, in patients with low risk tumors who are pN0. Bonnet et al. [8] reported that RAI was avoided in $15 \%$ of their patients with $\mathrm{T} 1$ tumors undergoing prophylactic neck dissection, who would have otherwise received RAI without documentation of pN0 status. This research group expanded on their cohort in a more recent publication in which $30 \%$ of their $\mathrm{T} 1$ and $\mathrm{T} 2$, pN0 patients did not receive radioiodine (though they also commented on a $26 \%$ rate of upstaging based on pN1 status) [9].

Whether changes in decisions regarding RAI administration based on prophylactic central nodal dissection have a long-term affect on outcomes for these patients remains to be seen. In our institution, we favor potential avoidance of RAI therapy, and currently offer ipsilateral central nodal dissection to cN0 patients with T1 or T2 tumors. We utilize the information from the nodal dissection to guide RAI decisions, specifically avoiding RAI in patients who are 
pNO without adverse tumor features. We feel that the tradeoff of potential surgical morbidity in the form of transient hypocalcemia is justified by potential avoidance of morbidity associated with RAI in this subset of patients, though we do not yet have long-term data regarding outcomes with this methodology and realize that this could lead to upstaging and potential overtreatment of some patients. As proposed in the 2012 ATA report by Randolph et al. [2], we hope that data from central dissections regarding number/size of positive nodes and extranodal extension could eventually be incorporated into a more detailed division of the $\mathrm{N}$ portion of the current AJCC TNM staging system to better risk stratify patients and plan RAI more judiciously.

Because a prospective randomized controlled trial regarding prophylactic neck dissection was deemed to be infeasible by the ATA in 2012 (due to the prohibitively large sample sizes required for sufficient statistical power) [10], this debate will likely continue. We anticipate further studies of institutional experience, similar to that reported here by Conzo et al. as well as ongoing expert review of this growing body of literature. It seems the future of prophylactic central nodal dissection studies lies in determining the actual significance of pathologically detected metastases and utilizing nodal dissection data to better risk stratify, deliver adjuvant treatment to, and plan follow-up for patients.

\section{References}

1. Conzo, G., Pasquali, D., Bellastella, G., Esposito, K., Carella, C., De Bellis, A., Docimo, G., Klain, M., Iorio, S., Napoliano, S., Palazzo, A., Pizza, A., Sinisi, A., Zampella, E., Bellastella, A., Santini, L., Total thyroidectomy, without prophylactic central lymph node dissection, in the treatment of differentiated thyroid cancer. Clinical retrospective study on 221 cases. Endocrine (2012)

2. G.W. Randolph, Q. Duh, K.S. Heller, V.A. LiVolsi, S.J. Mandel, D.L. Steward, R.P. Tufano, R.M. Tuttle, For the American
Thyroid Association Surgical Affairs Committee's Taskforce on Thyroid Cancer Nodal Surgery. The prognostic significance of nodal metastases from papillary thyroid carcinoma can be stratified based on the size and number of metastatic lymph nodes, as well as the presence of extranodal extension. Thyroid 22, 1144-1152 (2012)

3. W.T. Shen, L. Ogawa, D. Ruan, I. Suh, Q. Duh, O.H. Clark, Central neck lymph node dissection for papillary thyroid cancer: the reliability of surgeon judgment in predicting which patients will benefit. Surgery 148, 398-403 (2010)

4. M. Sywak, L. Cornford, P. Roach, P. Stalberg, S. Sidhu, L. Delbridge, Routine ipsilateral level VI lymphadenectomy reduces postoperative thyroglobulin levels in papillary thyroid cancer. Surgery 140, 1000-1007 (2006)

5. A. Popadich, O. Levin, J.C. Lee, S. Smooke-Praw, K. Ro, M. Fazel, A. Arora, N.S. Tolley, F. Palazzo, D.L. Learoyd, S. Sidhu, L. Delbridge, M. Sywak, M.W. Yeh, A multicenter cohort study of total thyroidectomy and routine central lymph node dissection for cN0 papillary thyroid cancer. Surgery 150, 1048-1057 (2011)

6. D.T. Hughes, M.L. White, B.S. Miller, P.G. Gauger, R.E. Burney, G.M. Doherty, Influence of prophylactic central lymph node dissection on postoperative thyroglobulin levels and radioiodine treatment in papillary thyroid cancer. Surgery 148, 1100-1107 (2010)

7. M. Shindo, J. Wu, E.E. Park, F. Tanzella, The importance of central compartment elective lymph node excision in the staging and treatment of papillary thyroid cancer. Arch. Otolaryngol. Head Neck Surg. 132, 650-654 (2006)

8. S. Bonnet, D. Hartl, S. Leboulleux, E. Baudin, J.D. Lumbroso, A. Al Ghuzlan, L. Chami, M. Schlumberger, J.P. Travagli, Prophylactic lymph node dissection for papillary thyroid cancer less than $2 \mathrm{~cm}$ : Implications for radioiodine treatment. J. Clin. Endocrinol. Metab. 94, 1162-1167 (2009)

9. D. Hartl, S. Leboulleux, A. Al Ghuzlan, E. Baudin, L. Chami, M. Schlumberger, J.P. Travagli, Optimization of staging of the neck with prophylactic central and lateral neck dissection for papillary thyroid carcinoma. Ann. Surg. 255, 777-783 (2012)

10. T. Carling, S.E. Carty, M.M. Ciarleglio, D.S. Cooper, G.M. Doherty, L.T. Kim, R.T. Kloos, E.L. Mazzaferri Sr, P.N. Peduzzi, S.A. Roman, R.S. Sippel, J.A. Sosa, B.C. Stack, D.L. Steward, R.P. Tufano, R.M. Tuttle, R. Udelsman, for the American Thyroid Association Surgical Affairs Committee. American Thyroid Association design and feasibility of a prospective randomized controlled trial of prophylactic central lymph node dissection for papillary thyroid carcinoma. Thyroid 22, 237-244 (2012)

11. L. Wartovsky, D. Van Nostrand, Radioiodine treatment of welldifferentiated thyroid cancer. Endocrine 42, 506-513 (2012) 